\title{
Inhibition of SIRT6 in prostate cancer reduces cell viability and increases sensitivity to chemotherapeutics
}

\author{
Yewei Liu ${ }^{1,2^{*}}$, Qian Reuben $X_{i e}{ }^{1^{*}}$, Boshi Wang ${ }^{1}$, Jiaxiang Shao ${ }^{1}$, Tingting Zhang ${ }^{1}$, Tengyuan Liu ${ }^{1}$, \\ Gang Huang ${ }^{2 \bowtie}$, Weiliang Xia ${ }^{1,3}$ \\ ${ }^{1}$ School of Biomedical Engineering and Med-X Research Institute, Shanghai Jiao Tong University, Shanghai 200030, China \\ ${ }^{2}$ Department of Nuclear Medicine, Renji Hospital, Shanghai Jiao Tong University School of Medicine, Shanghai 200127, China \\ ${ }^{3}$ Clinical Stem Cell Center, Renji Hospital, Shanghai Jiao Tong University School of Medicine, Shanghai 200127, China \\ $\triangle$ Correspondence: wlxia@sjtu.edu.cn (W. Xia), huang2802@163.com (G. Huang)
}

Received June 20, 2013 Accepted July 22, 2013

\begin{abstract}
SIRT6 is an important histone modifying protein that regulates DNA repair, telomere maintenance, energy metabolism, and target gene expression. Recently SIRT6 has been identified as a tumor suppressor and is downregulated in certain cancer types, but not in other cancers. From deposited gene profiling studies we found that SIRT6 was overexpressed in prostate tumors, compared with normal or paratumor prostate tissues. Tissue microarray studies confirmed the higher levels of SIRT6 in both prostate tumor tissues and prostate cancer cells than in their normal counterparts. Knockdown of SIRT6 in human prostate cancer cells led to sub- $\mathrm{G}_{1}$ phase arrest of cell cycle, increased apoptosis, elevated DNA damage level and decrease in BCL2 gene expression. Moreover, SIRT6-deficiency reduced cell viability and enhanced chemotherapeutics sensitivity. Taken together, this study provides the first evidence of SIRT6 overexpression in human prostate cancer, and SIRT6 regulation could be exploited for prostate cancer therapy.
\end{abstract}

KEYWORDS SIRT6, overexpression, prostate cancer, therapy

\section{INTRODUCTION}

Prostate cancer, which has the second highest morbidity and sixth highest mortality rate among all male cancer types worldwide, is a serious threat to men's health (Jemal et al., 2011). Treatment options for prostate cancer usually involve active

*These authors contributed equally to the work.

702 | September 2013 | Volume 4 | Issue 9 surveillance, surgery, radiation therapy, hormonal therapy and chemotherapy. Currently, hormone-refractory prostate cancer, which has been proved to be of heterogeneous morphology, immunophenotype, and genotype, remains a treatment challenge (Shah et al., 2004).

The sirtuin protein (SIRT1-7) family has been implicated in diverse physiological processes, as well as aging-associated diseases (Haigis and Sinclair, 2010). SIRT6 is predominantly concentrated in heterochromatic regions of the nucleus (Liszt et al., 2005; Michishita et al., 2005; Mostoslavsky et al., 2006), with conserved NAD-dependent protein deacetylase activity and ADP-ribosyltransferase ability (Liszt et al., 2005). SIRT6 is required for maintaining genomic stability (Xie et al., 2012; Cardus et al., 2013) and involved in base excision repair (BER) or DNA double-strand break (DSB) repair in response to genotoxic and oxidative stress (Kaidi et al., 2010). SIRT6 functions as a histone H3K9Ac deacetylase to repress the transcription factors HIF1 $\alpha, N F-K B$ and c-Jun and their target genes that are involved in apoptosis, inflammation, metabolism and senescence (Kawahara et al., 2009; Zhong et al., 2010; Kawahara et al., 2011; Sundaresan et al., 2012; Xiao et al., 2012). SIRT6 has been found to be therapeutically effective on age-related metabolic diseases largely through the influence on HIF1a target gene expression (Zhong et al., 2010) and IGF1R-IR/PI3K/ AKT/mTOR pathway regulation (Kawahara et al., 2011).

Cancer can be considered as an age-associated disease as well, with multiple hallmarks including sustained proliferative signaling, reprogramed energy metabolism and the involvement of inflammation (Hanahan and Weinberg, 2011). SIRT6 has been recently claimed to be a tumor suppressor due to observations that SIRT6 is downregulated in certain human 
cancers and SIRT6 deficiency causes increased glycolysis and tumor growth (Sebastian et al., 2012). Accordantly, overexpression of SIRT6 in multiple cancer cells induces apoptosis mediated by p53 and p73 signaling pathway (Van Meter et al., 2011). At the initiation stage of liver cancer, c-Fos induces SIRT6 transcription, which inhibits the activity of survivin and impairs cancer development (Min et al., 2012). However, it was shown recently that, in pancreatic cancer, SIRT6 promotes proinflammatory cytokines expression through regulation of $\mathrm{Ca}^{2+}$ responses (Bauer et al., 2012). How SIRT6 regulates cancer development in different tissue types possibly relates to the cellular context and origin of the cancers. For prostate cancer, the role of SIRT6 remains undetermined.

In our study, we have used both prostate tumor tissues and prostate cancer cell lines to study the roles of SIRT6. Following the detection of a higher level of SIRT6 in prostate tumor tissues and prostate cancer cell lines, we used PC- 3 and DU145 cells as a model to study the effects of SIRT6 knockdown on the cancer cells with respect to cell proliferation, cell cycle, apoptosis, DNA damage level, BCL2 gene expression and resistance to chemotherapeutics. Moreover, Kaplan-Meier analysis of the data archived in Oncomine indicated that high SIRT6 expression associated with unfavorable overall survival and recurrence-free survival (Taylor et al., 2010). Our findings uncover some of the functions of SIRT6 that are essential for prostate cancer progression, and might provide a new approach on prostate cancer therapy.

\section{RESULTS}

\section{SIRT6 overexpression in both prostate tumor tissues and prostate cancer cell lines}

To investigate the role of SIRT6 in prostate cancer progression, we queried the NCBl's GEO datasets. Microarray analysis of prostate cancer profiles (GSE6919) revealed that SIRT6 was higher expressed in metastatic prostate tumors, compared with normal or paratumor prostate tissues or even primary prostate tumors (Figs. 1A and S1). In Oncomine database (http://www. oncone.org), the similar results were found (Taylor et al., 2010) that SIRT6 mRNA level was higher in prostate carcinoma than in prostate gland (Fig. 1B). We examined SIRT6 expression on prostate cancer tissue arrays with 25 paired human prostate cancer tissues and corresponding prostate tissues. Highest level of SIRT6 was detected in the nucleoplasm of prostate cancer epithelial cells, with a diffusive pattern that also permeated the cytoplasm of these cells. Lower level of SIRT6 was also found to be scattered in the interstitial tissues (Fig. 1C). All in all, the immunohistochemical analyses showed there was a significantly higher level of SIRT6 in prostate tumor tissues compared with paired paratumor tissues in the prostate cancer tissue arrays (Fig. 1C and 1D). We then screened the levels of SIRT6 protein in different prostate cancer cell lines (PC-3, DU145, 22RV1 and LNCaP), together with the human benign prostate hyperplasia cell line (BPH-1) and normal prostatic epi- thelial cell line (RWPE-1) by Western blot analysis. SIRT6 level was significantly higher in prostate cancer cells than in benign prostate hyperplasia cells and in normal prostatic epithelial cells (Fig. 1E).

To test the correlation of SIRT6 expression with survival of postoperative prostate cancer patients, we performed a comprehensive analysis of SIRT6 expression from the previously published database in Oncomine (Taylor et al., 2010). Survival data were available for 138 patients. The average survival time for the patient with SIRT6 high expression was 64 months, while for the SIRT6 low expression was 104 months. The Kaplan-Meier survival analyses showed that patients with high SIRT6 expression were likely to have shorter overall survival $(P<0.0001)$ and recurrence-free survivals $(P<0.0001)$ (Fig. 2$)$.

Down-regulation of SIRT6 expression reduces cell growth and induces apoptosis in prostate cancer cells

To further investigate the biological role of SIRT6 in the prostate cancer cells, we used RNAi approach to repress SIRT6 expression in prostate cancer cell lines PC-3 and DU145. Both SIRT6 and SIRT1 have been shown to be predominantly located in the nucleus (Michishita et al., 2005), attenuate NF-KB signaling pathways (Kawahara et al., 2009) and repress HIF1 $\alpha$ transcriptional activity (Zhong et al., 2010). In order to confirm that SIRT6 silencing was effective and selective, Western blot analysis of SIRT6 and SIRT1 protein levels in SIRT6 knockdown cells and negative control (NC siRNA) cells was performed. SIRT6 protein level but not SIRT1 was reduced both in PC-3 and DU145 cells (Fig. 3A). We then tested how the reduced level of SIRT6 in prostate cancer cells would affect cellular functions. PC-3 and DU145 cells were cultured and transfected with either NC siRNA or SIRT6 siRNA, and cell number was counted. SIRT6-knockdown in both cell lines reduced cell number by $30 \%$, compared with NC siRNA treated cells (Fig. 3B). This suggested that SIRT6 knockdown could ultimately reduce cell growth. Cell cycle analysis showed a reduction of cell population at $G_{2} / M$ phase and an increase at the sub- $G_{1}$ phase in PC-3 cells after down-regulation of SIRT6 (Fig. 3C). This SIRT6 knockdown-induced sub- $\mathrm{G}_{1}$ phase arrest was indicative of apoptosis. The flow cytometric apoptosis assay was followed using an Annexin V/7-AAD kit in PC-3 and DU145 cells. SIRT6 deficiency also increased the cell apoptosis rates compared with the negative control cells $(\sim 5.6 \%$ and $\sim 8.9 \%$ in NC and SIRT6 siRNA cells of PC-3; $\sim 3.7 \%$ and $\sim 4.8 \%$ in NC and SIRT6 siRNA cells of DU145, Fig. 3D).

Increased DNA damage and reduced BCL2 expression are involved in SIRT6 related cell death

To examine whether increased DNA damage sensitivity is associated with the elevated apoptosis, DNA double-strand breaks were induced by Paraquat and quantified by $\mathrm{y}-\mathrm{H} 2 \mathrm{AX}$ staining in the NC siRNA or SIRT6 siRNA transfected DU145 cells (Fig. 4A and 4B). Consistent with the observed increases 

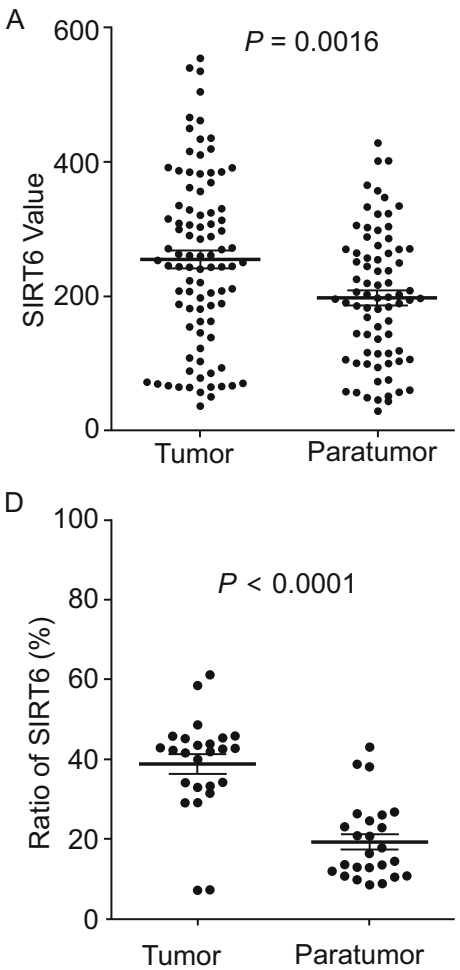

B

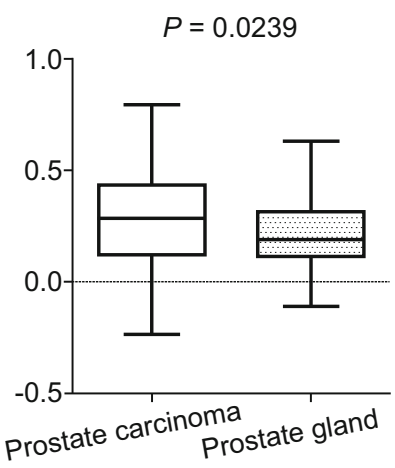

$\mathrm{E}$
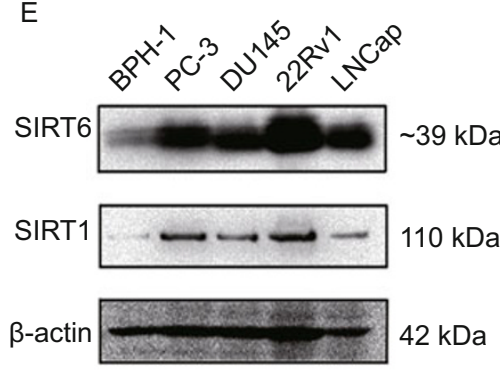

C

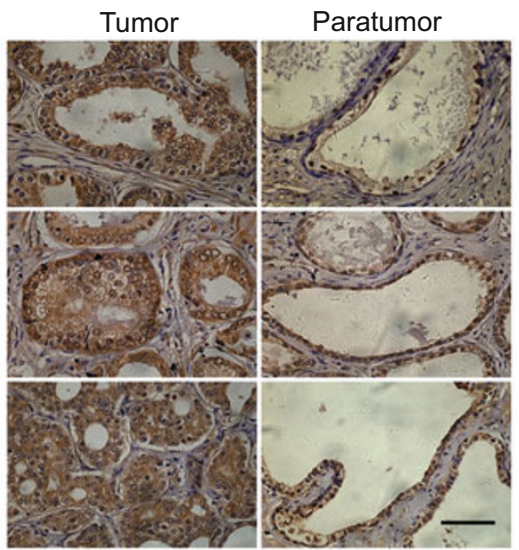

Figure 1. Overexpression of SIRT6 in human prostate tumor/paratumor tissues and prostate cancer cells. (A) Dot plot represented SIRT6 expression value from microarray analysis of prostate cancer profiles retrieved from NCBI database (GSE6919). The samples were divided into two groups: tumor tissue group (left panel) including primary prostate tumor $(n=66)$ and metastatic prostate tumor $(n=25)$; paratumor tissue group (right panel) including normal prostate issue $(n=17)$, normal prostate adjacent to tumor $(n=59)$. The $P$ value $=0.0016$ was determined by paired Student's $t$ test. $(B)$ Microarray expression of the prostate cancer database was downloaded from Oncomine (http://www.oncomine.org) to examine the relative expression of SIRT6 between normal (prostate gland, $n=29$ ) and cancer (prostate carcinoma, $n=131$ ) samples. The distribution of $\log 2$ median-centered signal intensities was plotted using boxplots. (C) SIRT6 was immunostained in both human prostate tumor (left panel) and paired paratumor (right panel) tissues. Representative pictures are shown. The scale bar represents $50 \mu \mathrm{mol} / \mathrm{L}$. (D) Dot plot represented SIRT6 ratio from each of the tumor and paratumor tissues $(n$ = 25 pairs of samples). SIRT6 ratio was evaluated as the percentage of area positively stained versus total area stained (empty space was excluded), analyzed independently by two pathology specialist using Leica $Q$ Win software. The $P$ value was determined by paired Student's $t$ test with a value $<0.0001$. (E) Comparison of SIRT6 protein expressions in prostate cancer cell lines (PC-3, DU145, 22Rv1, $\mathrm{LNCaP})$, benign prostate hyperplasia cell line $(\mathrm{BPH}-1)$ and normal prostatic epithelial cell line (RWPE-1) were detected by western blotting. SIRT1 protein expression was detected as well; $\beta$-actin and $\alpha$-Tubulin were used as the loading controls.

in apoptosis, we found an increase in $\mathrm{Y}-\mathrm{H} 2 \mathrm{AX}$ positive foci in the SIRT6 knockdown cells. After Paraquat exposure, SIRT6 siRNA transfected cells displayed significantly more $\mathrm{Y}-\mathrm{H} 2 \mathrm{AX}$ positive foci (Fig. 4A and 4B), which indicated that SIRT6 deficiency rendered these cells more sensitive to DNA damage. On the other hand, mRNA and protein levels of apoptosis-related BCL2 were examined by real-time PCR and Western blot. These analyses showed the levels of BCL2 were decreased both in PC-3 and DU145 cells after SIRT6 silencing (Fig. 4C and 4D).

\section{Down-regulation of SIRT6 expression enhances chemotherapeutics sensitivity}

To test whether SIRT6 inhibition could increase chemotherapy sensitivity, CCK-8 assay was used to examine cell viability, which indicated that SIRT6 knockdown induced $\sim 13 \%$ and $\sim 11 \%$ decrease in the viability of SIRT6 silenced PC-3 and DU145 cells, respectively. However, after $24 \mathrm{~h}$ treatment with Taxol, SIRT6 deficiency decreased the cell viability in DU145 cells $(\sim 83 \%$ and $\sim 68 \%$ in NC siRNA and SIRT6 siRNA treated cells, respectively, Fig. $5 \mathrm{~A}$, right), even though the decreased tendency did not show statistical significance in PC-3 cells $(\sim 72 \%$ and $\sim 67 \%$ in NC and SIRT6 siRNA treated cells, respectively, Fig. 5A, left). Using the Annexin V/7-AAD apoptosis flow cytometirc measurement, it was found that apoptosis was increased in PC-3 and DU145 SIRT6 silencing cells treated with Taxol for $24 \mathrm{~h}$ (Fig. 5B). Taken together, SIRT6 silencing resulted in chemo drug (Taxol) sensitization in PC-3 and DU145 cells. 

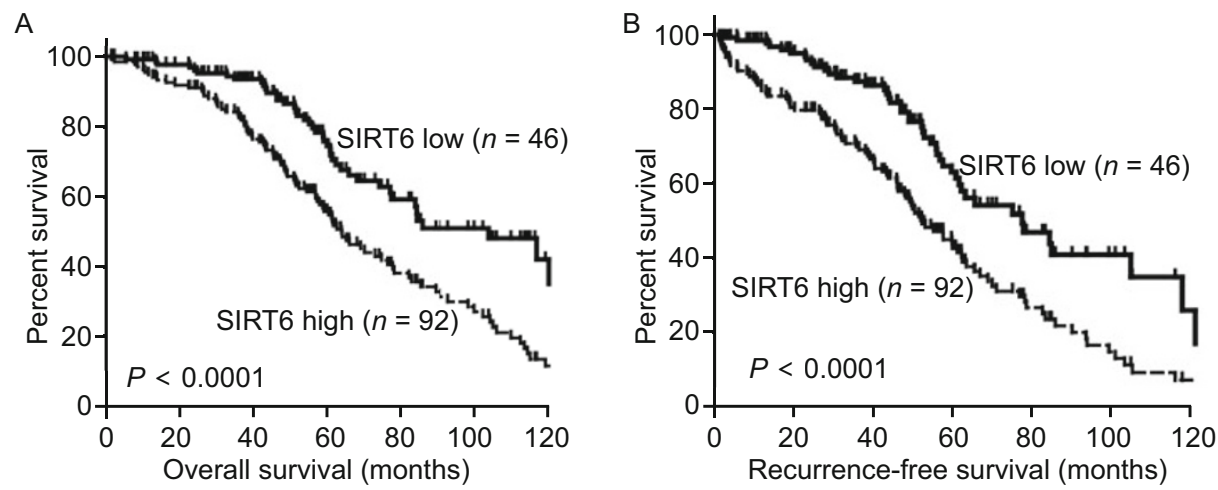

Figure 2. Correlation of SIRT6 high expression in prostate cancer tissue with unfavorable overall survival and recurrence-free survival from the bioinformatics analysis. Probabilities of overall survival (A) and recurrence-free survival (B) of total 138 prostate cancer patients from the database in Oncomine were analyzed by Kaplan-Meier survival analysis (log-rank test) comparing with high $(>50$ th percentile) versus low ( $<50$ th percentile) SIRT6 expression.

A

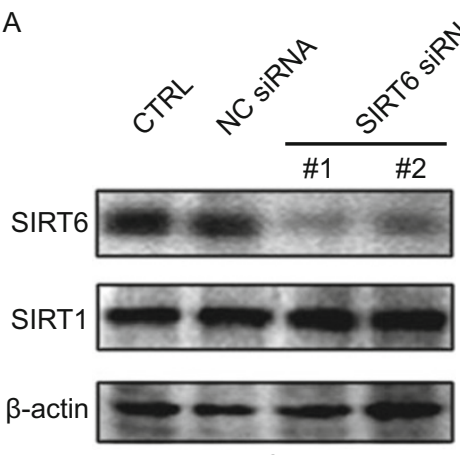

PC-3<smiles>O=S</smiles><smiles>C1CCCCC1</smiles>

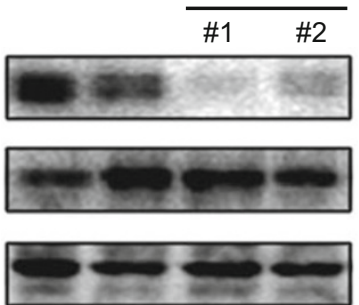

DU145

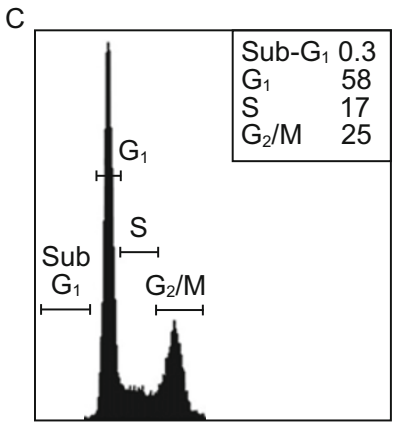

NC SiRNA

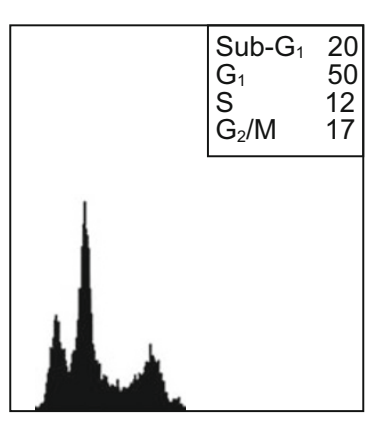

SIRT6 SIRNA

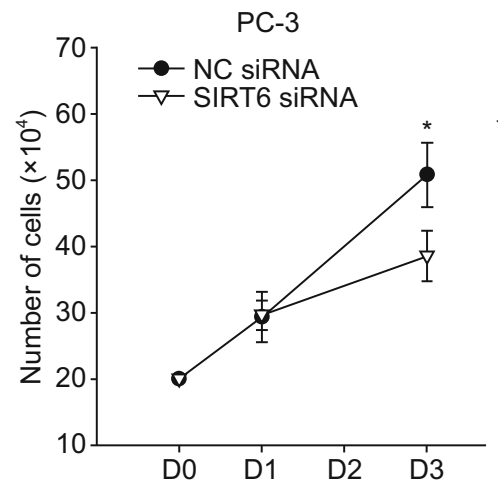

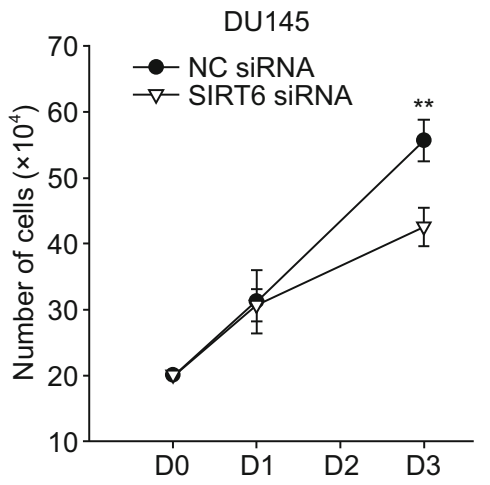

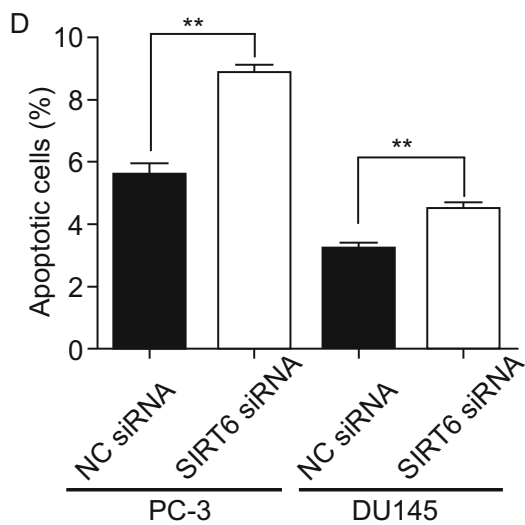

Figure 3. Downregulation of SIRT6 in human prostate cancer cell lines and effect of SIRT6 knockdown on cell growth, cell cycle progression and apoptosis. (A) PC-3 (left panel) and DU145 (right panel) cells were transfected with SIRT6 (Target sequence 1 and 2: \#1, \#2) siRNAs or negative control (NC) siRNA, as described in Materials and Methods. The expression of SIRT6, SIRT1 and $\beta$-actin was detected by Western blotting, and representative images were shown from three independent experiments. (B) Cell proliferation of SIRT6 knockdown cells $(\nabla)$ and negative control cells $(\bullet)$ was assessed by direct cell counting. PC-3 (left) and DU145 (right) cells were seeded at a density of $2 \times 10^{5}$ cells in a 6-well plate, counted on the day of transfection (D1) and 2 days after transfection (D3). (C) SIRT6 knockdown cells and negative control cells were stained with PI for cell cycle profile analysis by flow cytometry. The percentage of cell population in each cell cycle phase was included in the inset of the graph. (D) Quantification of apoptosis analysis using Annexin V/7-AAD staining by flow cytometry. The apoptotic cells (\%) indicated the percentage of cells undergoing early (Annexin $\mathrm{V}^{+} / 7-\mathrm{ADD}^{-}$) and late (Annexin $\left.\mathrm{V}^{+} / 7-\mathrm{AAD}^{+}\right)$apoptosis. Values are expressed as the mean $\pm \mathrm{SEM}\left({ }^{*}, P<0.05\right.$; $\left.{ }^{* *}, P<0.01\right)$ of three independent experiments. 
A
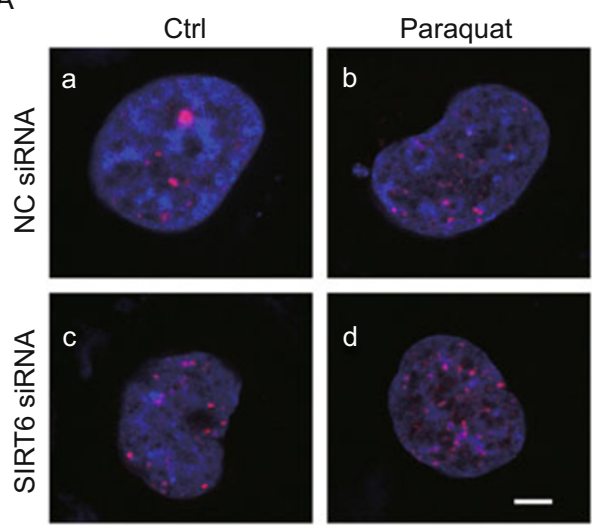

C

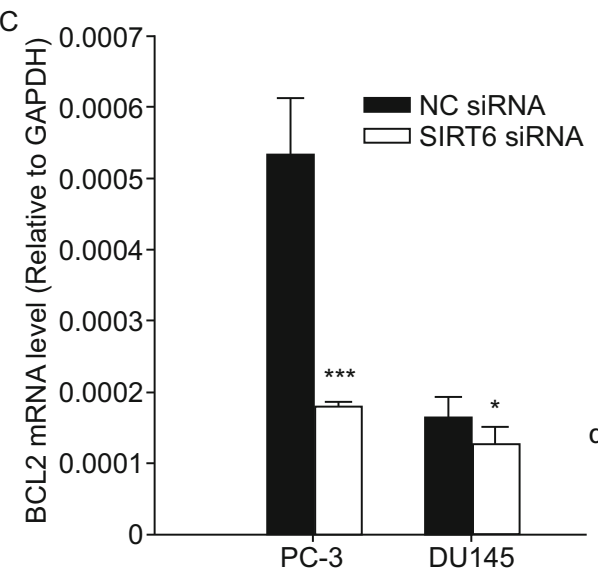

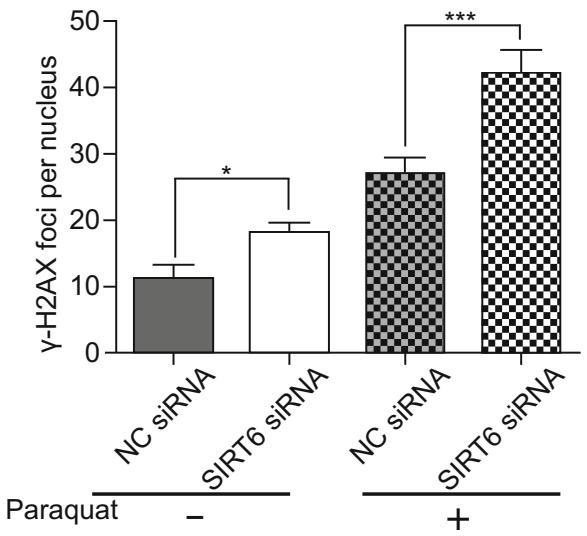

D

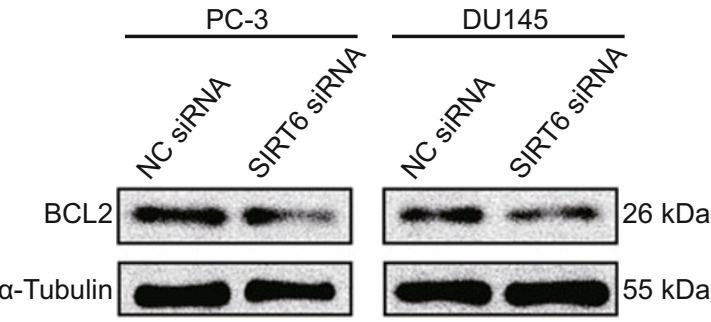

Figure 4. Effects of SIRT6 knockdown on DNA damage and BCL2 expression. (A) Representative images of NC (NC siRNA), SIRT6 (SIRT6 siRNA)-depleted DU145 cells that were treated with $100 \mathrm{mmol} / \mathrm{L}$ Paraquat for $2 \mathrm{~h}$ and immunostained with antibodies against $\mathrm{Y}-\mathrm{H} 2 \mathrm{AX}$ (red) and counterstained with DAPI (blue). The scale bar represents $15 \mu \mathrm{m}$. (B) Quantification of $\mathrm{Y}-\mathrm{H} 2 \mathrm{AX}$ foci per cell. ( $n=25-30$ cells analyzed per condition with at least two independent experiments). The mRNA expression of BCL2 (C) and its protein level (D) in PC-3 and DU145 cells after being transfected with SIRT6 siRNA or NC siRNA for $48 \mathrm{~h}$. Data were normalized to GAPDH and were expressed as relative expression levels. $\alpha$-Tubulin was used as the loading control. Values are expressed as mean $\pm \mathrm{SEM}\left({ }^{*}, P<0.05\right.$; ${ }^{* *}$, $P<0.01 ;{ }^{* * *}, P<0.001$ ) from three independent experiments.

\section{DISCUSSION}

In this study, we have reported for the first time that SIRT6 is specifically overexpressed in both prostate cancer tissues and cell lines (Fig. 1). Down-regulation of SIRT6 in prostate cancer cells was shown to affect prostate cancer cells via the following effects including increased apoptosis (Fig. 3), reduced cell viability (Fig. 5), which could be associated with elevated DNA damage level and repressed BCL2 gene expression (Fig. 4). And, remarkably, SIRT6 repression enhances chemotherapeutics sensitivity in prostate cancer cells (Fig. 5).

Regarding the function of SIRT6 in cancer, different points of view are held. SIRT6 is claimed to be a tumor repressor based on the observations that SIRT6 is downregulated in certain human cancers and SIRT6 deficiency causes increased glycolysis and tumor growth (Sebastian et al., 2012). Consistently, SIRT6 overexpression stimulates apoptosis in cervical carcinoma, fibrosarcoma and breast cancer cells, but not in the normal cells, under the hypothesis that SIRT6 was a tumor repressor (Van Meter et al., 2011). However, we observed that in prostate cancer, SIRT6 protein expression was significantly increased in the malignant prostate tissues and cells. Deficiency of SIRT6 in PC-3 and DU145 cells suppressed cell growth (Fig. 3B), viability (Fig. 5A) and enhanced apoptosis (Fig. 3D), suggesting that SIRT6 may promote prostate cancer and serve as a therapeutic target. In the heart tissue of Sirt6 knockout mice, increased expression of apoptotic markers such as Caspase-3, Bax, TRAIL, Bim, FasL and p27 was observed (Sundaresan et al., 2012). Nevertheless, the details of mechanisms involved in SIRT6-downregulation-driven apoptosis in prostate cancer remain to be unraveled. Chronic inflammation is associated with cancer progression (Hanahan and Weinberg, 2011). In the recent study on pancreatic cancer, SIRT6 promotes pro-inflammatory cytokines expression (Bauer et al., 2012). In our prostate cancer cell culture models, repression of SIRT6 also reduced pro-inflammation cytokines expression 
A

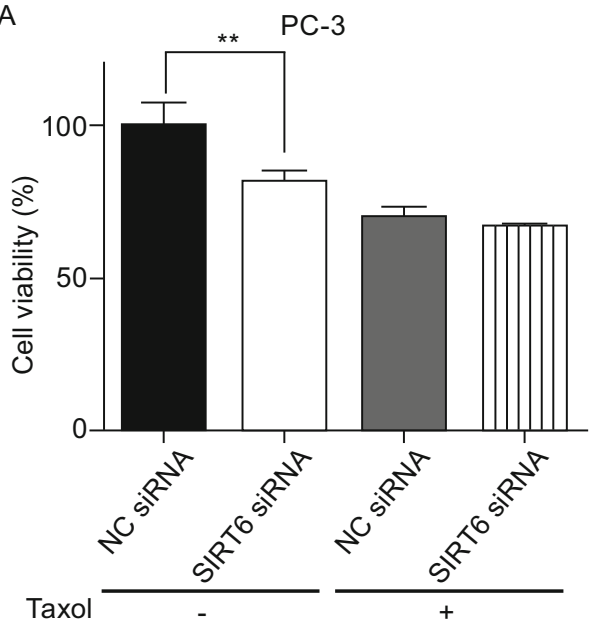

B

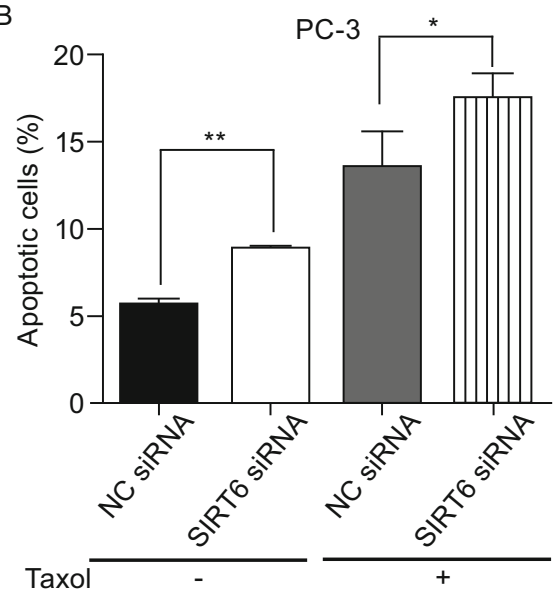

DU145

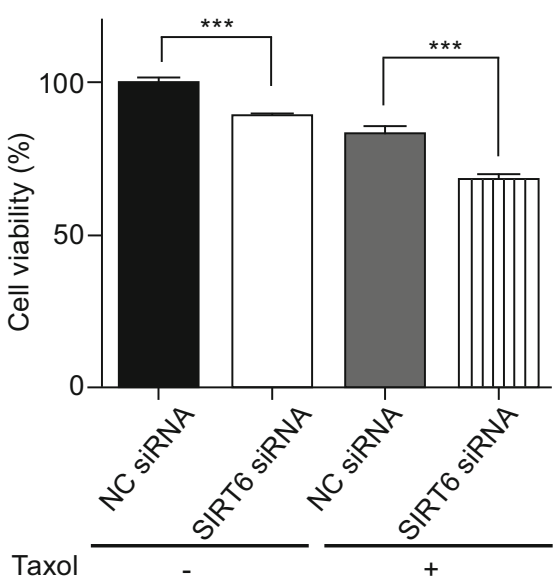

DU145

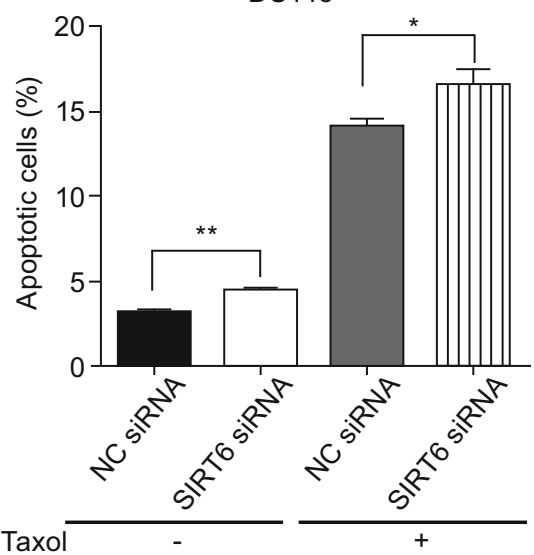

Figure 5. Effect of SIRT6 knockdown on cell viability/apoptosis and chemo sensitivity. (A) SIRT6-knockdown PC-3 and DU145 cells were seeded into 96 -well plates at the density of $5 \times 10^{3}$ cells per well for $24 \mathrm{~h}$, and exposed to $100 \mathrm{nmol} / \mathrm{L}$ Taxol or $0.017 \%$ DMSO for another $24 \mathrm{~h}$. Then the cell viability was detected. (B) Cell apoptosis induced by SIRT6 knockdown and/or Taxol treatment was quantified using Flow Cytometric Annexin V/7-AAD analysis. Cells were plated in 6-well plates at the density of $2 \times 10^{5}$ cell per well for $24 \mathrm{~h}$, transfected with either SIRT6 siRNA or negative control siRNA for $48 \mathrm{~h}$, and exposed to $100 \mathrm{nmol} / \mathrm{L}$ Taxol or $0.017 \%$ DMSO for another $24 \mathrm{~h}$. Then cells were harvested, stained by the Annexin V/7-AAD kit, and analyzed by flow cytometry for cell death. Values are expressed as the mean \pm SEM $\left({ }^{*}, P<0.05 ;{ }^{* *}, P<0.01 ;{ }^{* * *}, P<0.001\right)$ from three independent experiments.

(data not shown). SIRT6 is specifically overexpressed in the initiation stage of liver cancer, but not in the advanced liver cancer (Min et al., 2012). High SIRT6 expression has been identified as a poor prognostic factor for both overall survival and recurrence-free survival in prostate cancer patients from our analysis of SIRT6 expression in the previously published data from Oncomine (Taylor et al., 2010). The high SIRT6 expression was associated with markedly shorter period of clinical recurrence (Fig. 2). Khongkow's work revealed that high SIRT6 nuclear expression was associated with poor survival in the breast cancer patients (Khongkow et al., 2013). However, low SIRT6 expression was reported to connect to poor survival in hepatocellular carcinoma (Marquardt et al., 2013). The clinical significance of SIRT6 in prostate cancer is worthy of further investigation by analyzing a large number of prostate tumor samples at different stages to explore the different role of SIRT6 in the prostate cancer from that in the hepatocellular carcinoma. It is possible that SIRT6's function in malignancy varies with the location, concentration, distribution and the regulation by upstream and downstream factors, similar to the role of SIRT1 in carcinogenesis (Bosch-Presegue and Vaquero, 2011; Song and Surh, 2012). The controversy over whether SIRT6 serves as a tumor promoter or a tumor suppressor has not been completely resolved and the discussion will likely continue.

Radiotherapy is known as a routine treatment for local prostate cancer at the early stage. Downregulation of SIRT6 can enhance sensitivity to radiation damage in multiple cells lines including MEF and WI-38 (Mostoslavsky et al., 2006; McCord et al., 2009). Since SIRT6 is essential for genomic stability, 
radiation-induced damage in SIRT6-deficient cancer cells might be more pronounced that could lead to greater sensitivity for radiation therapy. This hypothesis will also require further investigation.

In conclusion, our data suggest that SIRT6 protein is overexpressed in both prostate tumor tissues and prostate cancer cells compared to their normal counterparts. Knockdown of SIRT6 in human prostate cancer cells leads to sub- $G_{1}$ phase arrest of cell cycle, increased apoptosis possibly via elevated DNA damage and alteration of BCL2 gene expression. Moreover, SIRT6-deficiency reduces prostate cancer cell viability and enhances chemotherapeutics sensitivity.

\section{MATERIALS AND METHODS}

Antibodies and reagents

The primary antibodies were as follows: SIRT6 (ab62738, 1:1000, Abcam); BCL2 (\#1017-1, 1:500, Epitomics Inc); SIRT1 (sc-15404, 1:500, Santa Cruz Biotechnology); $\beta$-actin (sc-1616, 1:1000, Santa Cruz Biotechnology); $\alpha$-Tubulin (T5168, 1:2000, Sigma-Aldrich Inc). The secondary antibodies were purchased from HuaAn Biotechnology (Hangzhou, China). Paclitaxel and Paraquat were purchased from Sigma-Aldrich (St. Louis, MO, USA). All other reagents were obtained from Sigma-Aldrich (St. Louis, MO, USA).

\section{Cell culture}

Human prostate carcinoma cell lines (PC-3, DU145, 22RV1 and LN$\mathrm{CaP}$ ) were purchased from the Cell Resource Center of Shanghai Institute of Biological Sciences, Chinese Academy of Sciences. Human prostatic epithelial cell line (RWPE-1) and benign prostatic hyperplasia cell line (BPH-1) was a gift from Dr. Wei-Qiang Gao (Clinical Stem Cell Center, Renji Hospital). Cells were maintained in RPMI 1640 medium (Hyclone, Logan, UT, USA) supplemented with $10 \%$ fetal bovine serum and $1 \%$ penicillin/streptomycin (Invitrogen, Carlsbad, CA, USA) at $37^{\circ} \mathrm{C}$ in a humidified incubator under $5 \% \mathrm{CO}_{2}$.

\section{RNAi}

PC3 and DU145 cells at $60 \%-70 \%$ confluency were transfected with $50 \mathrm{nmol} / \mathrm{L}$ siRNA using Lipofectamine 2000 (Invitrogen, Carlsbad, CA, USA) according to manufacturer's protocols. Target sequence 1 (\#1) for SIRT6: 5'-GAAUGUGCCAAGUGUAAGATT-3'; Target sequence 2 (\#2) for SIRT6: 5'-CCGGCUCUGCACCGUGGCUAATT-3'; Target sequence for negative control: 5'-CGACAUACUGUACAGGCCUTT-3'.

\section{Western blot}

Whole cell proteins were lysed in RIPA buffer (Millipore, Temecula, CA, USA) containing Complete Protease Inhibitor Cocktail, $2 \mathrm{mmol} / \mathrm{L}$ PMSF, and $0.1 \%$ SDS. The protein concentration was measured using BCA assay kit (Thermo Scientific, Rockford, IL, USA). $30 \mu \mathrm{g}$ of total protein was separated by $8 \%-10 \%$ SDS-PAGE and then transferred to $0.45 \mu \mathrm{m}$ Nitrocellulose Membrane (Millipore, CA, USA). The membrane was blocked with TBST containing 5\% non-fat milk, incubated with primary antibodies at $4^{\circ} \mathrm{C}$ overnight and then hybridized with appropriate HRP-conjugated secondary antibody at room temperature for $1 \mathrm{~h}$. Protein signals were visualized using ECL detection system
(Thermo Scientific, Rockford, IL, USA).

Tissue microarray and immunohistochemical staining

SIRT6 expression levels were examined using prostate adenocarcinoma tissue microarray (Shanghai Outdo Biotech Co. Ltd, ODCT-UrPrt03), containing 29 pairs of prostate cancer and adjacent noncancerous tissues. All of the specimens were formalin-fixed and embedded in paraffin. Immunohistochemical staining was carried out using Histostain-Plus Kit (\#95-6143, Invitrogen, Carlsbad, CA, USA). In brief, specimen sections were deparaffinized in xylene, rehydrated in alcohol, treated with $3 \%$ hydrogen peroxide for $10 \mathrm{~min}$ and blocked with $10 \%$ BSA in PBS for 30 min. Sections were incubated with SIRT6 antibody (ab62738, 1:150, Abcam) at $4^{\circ} \mathrm{C}$ overnight, hybridized with biotinylated secondary antibodies for $1 \mathrm{~h}$, and then with HRP-streptavidin complex for $15 \mathrm{~min}$. Color was developed using an HRP substrate and nuclei were counterstained with hematoxylin. SIRT6 expression in prostatic tumor and paratumor tissues was evaluated as the percentage of positively stained area versus total stained area (empty space was excluded), analyzed independently by two pathology specialist using Leica QWin software.

\section{Immunofluorescence staining}

The immunofluorescence staining for $\mathrm{Y}-\mathrm{H} 2 \mathrm{AX}$ was performed as previously described (Xie et al., 2013). Cells were cultured on coverslips and washed twice with cold PBS, fixed with $4 \%$ paraformaldehyde for $30 \mathrm{~min}$, permeabilized with $0.1 \%$ Triton $\mathrm{X}-100$ for $15 \mathrm{~min}$, and blocked with $5 \%$ normal donkey serum for $1 \mathrm{~h}$. Cells were then incubated with antibody against $\mathrm{Y}-\mathrm{H} 2 \mathrm{AX}$ (\#05-636, 1:200, Millipore, Billerica, MA, USA) for $4 \mathrm{~h}$, washed, and incubated with AlexaFluor 594 secondary antibody (Molecular Probes, Eugene, OR, USA). Cells were mounted with VectaShield Mounting media with DAPI nuclear stain (Vector Labs, Burlingame, CA, USA). Fluorescence images were taken using a Leica SP5II Confocal Microscope (Leica Microsystem, Germany).

\section{Cell cycle analysis by flow cytometry}

Transfected PC-3 cells were harvested, washed twice with PBS and fixed in $70 \%$ ethanol at $4^{\circ} \mathrm{C}$ overnight. After being washed, the cells were incubated in PBS containing $40 \mu \mathrm{g} / \mathrm{mL}$ propidium iodide, $100 \mu \mathrm{g} / \mathrm{mL}$ RNaseA and $0.1 \%$ Triton $\mathrm{X}-100$ at $37^{\circ} \mathrm{C}$ for $30 \mathrm{~min}$. DNA content was measured by Quanta SC Flow Cytometer (Beckman Coulter, Miami, FL, USA), and cell cycle analysis was performed using MultiCycle software (Phoenix Flow Systems).

\section{Apoptosis analysis by flow cytometry}

Apoptosis was evaluated using the Annexin V/7-AAD Apoptosis Detection Kit (Southern Biotechnology, Birmingham, AI, USA). Briefly, transfected PC-3 and DU145 cells were harvested, washed twice with PBS, resuspended in binding buffer, stained with Annexin $V$ and 7-AAD on ice for $15 \mathrm{~min}$, and subjected to flow cytometer (BD FACSAriall, San Jose, CA, USA).

\section{Cell proliferation assay}

PC-3 and DU145 cells were seeded at a density of $2 \times 10^{5}$ cells in a 6-well plate and transfected with siRNA. $24 \mathrm{~h}$ later, cells were har- 
vested and counted directly on the day of transfection and 2 days after transfection.

\section{CCK-8 cell viability assay}

Cell viability was measured by using Cell Counting Kit-8 (Dojindo Laboratories, Kumamoto, Japan). In brief, cells were seeded in 96-well plates at the density of $5 \times 10^{3}$ per well. After treatment, CCK8 solution was added to the medium at a dilution of $1: 10$ and incubated at $37^{\circ} \mathrm{C}$ for $1-3 \mathrm{~h}$. The absorbance at $450 \mathrm{~nm}$ was determined using a microplate reader (Synergy2, BioTek, Winooski, VT, USA).

\section{Real-time quantitative PCR}

Total RNA was isolated from PC-3 and DU145 cells by Trizol Reagent and reverse-transcribed to cDNA using PrimeScript RT reagent kit (TaKaRa, Japan). Quantitative PCR was performed on ABI 7900HT by using SYBR Premix Ex Taq (TaKaRa, Japan) and the following primers: BCL2 (Forward: 5'-GGGGAGGATTGTGGCCTTC-3' and reverse: 5'-CAGGGCGATGTTGTCCACC-3'); GAPDH (Forward: 5'-GCGACCTGGAAGTCCAACTAC-3' and reverse: 5'-ATCTGCTGCATCTGCTTGG-3'). A cycle threshold was determined for each gene of interest and normalized to housekeeping gene (GAPDH) determined in parallel.

\section{Statistical analysis}

Each experiment was repeated at least three times. All data were presented as mean \pm SEM and assessed by one-way ANOVA, followed by Tukey post hoc test. $P$ values less than 0.05 were considered statistically significant.

\section{ACKNOWLEDGMENTS}

The study was supported by research grants from National Natural Science Foundation of China (Grant Nos. 30830038, 30970842, 81071180, 30900756 and 31270032), the National Basic Research Program (973 Program) (No. 2012CB932604), New Drug Discovery Project (2012ZX09506-001-005), Key Project of Science and Technology Commission of Shanghai Municipality (No. 10JC1410000), Shanghai Leading Academic Discipline Project (No. S30203); Visiting Scholarship for PhD student of SJTU to QRX. We are grateful to Ms. Jin Xu for her assistance in flow cytometry, Ms. Lin Zheng for her assistance in tissue array statistic work and Mr. Jian Yang for his assistance in paper proofing.

\section{COMPLIANCE WITH ETHICS GUIDELINES}

All authors declare no conflict of interests. This article does not contain any studies with human or animal subjects performed by the any of the authors.

\section{REFERENCES}

Bauer, I., Grozio, A., Lasiglie, D., Basile, G., Sturla, L., Magnone, M., Sociali, G., Soncini, D., Caffa, I., Poggi, A., et al. (2012). The NAD+dependent histone deacetylase SIRT6 promotes cytokine production and migration in pancreatic cancer cells by regulating $\mathrm{Ca} 2+$ responses. J Biol Chem 287, 40924-40937.

Bosch-Presegue, L., and Vaquero, A. (2011). The dual role of sirtuins in cancer. Genes Cancer 2, 648-662.

Cardus, A., Uryga, A.K., Walters, G., and Erusalimsky, J.D. (2013). SIRT6 protects human endothelial cells from DNA damage, telomere dysfunction, and senescence. Cardiovasc Res 97, 571-579.

Haigis, M.C., and Sinclair, D.A. (2010). Mammalian sirtuins: biological insights and disease relevance. Ann Rev Pathol 5, 253-295.

Hanahan, D., and Weinberg, R.A. (2011). Hallmarks of cancer: the next generation. Cell 144, 646-674.

Jemal, A., Bray, F., Center, M.M., Ferlay, J., Ward, E., and Forman, D. (2011). Global cancer statistics. CA: a cancer journal for clinicians 61, 69-90.

Kaidi, A., Weinert, B.T., Choudhary, C., and Jackson, S.P. (2010). Human SIRT6 promotes DNA end resection through CtIP deacetylation. Science 329, 1348-1353.

Kawahara, T.L., Michishita, E., Adler, A.S., Damian, M., Berber, E., Lin, M., McCord, R.A., Ongaigui, K.C., Boxer, L.D., Chang, H.Y., et al. (2009). SIRT6 links histone H3 lysine 9 deacetylation to NFkappaB-dependent gene expression and organismal life span. Cell $136,62-74$

Kawahara, T.L., Rapicavoli, N.A., Wu, A.R., Qu, K., Quake, S.R., and Chang, H.Y. (2011). Dynamic chromatin localization of Sirt6 shapes stress- and aging-related transcriptional networks. PLoS Genet 7 , e1002153.

Khongkow, M., Olmos, Y., Gong, C., Gomes, A.R., Monteiro, L.J., Yague, E., Cavaco, T.B., Khongkow, P., Man, E.P., Laohasinnarong, S., et al. (2013). SIRT6 modulates pa epirubicin resistance and survival in breast cancer. Carcinogenesis. 34, 1476-1486.

Liszt, G., Ford, E., Kurtev, M., and Guarente, L. (2005). Mouse Sir2 homolog SIRT6 is a nuclear ADP-ribosyltransferase. J Biol Chem 280, 21313-21320.

Marquardt, J.U., Fischer, K., Baus, K., Kashyap, A., Ma, S., Krupp, M., Linke, M., Teufel, A., Zechner, U., Strand, D., et al. (2013). SIRT6 dependent genetic and epigenetic alterations are associated with poor clinical outcome in HCC patients. Hepatology. (In Press).

McCord, R.A., Michishita, E., Hong, T., Berber, E., Boxer, L.D., Kusumoto, R., Guan, S., Shi, X., Gozani, O., Burlingame, A.L., et al. (2009). SIRT6 stabilizes DNA-dependent protein kinase at chromatin for DNA double-strand break repair. Aging 1, 109-121.

Michishita, E., Park, J.Y., Burneskis, J.M., Barrett, J.C., and Horikawa, I. (2005). Evolutionarily conserved and nonconserved cellular localizations and functions of human SIRT proteins. Mol Biol Cell 16, 4623-4635.

Min, L., Ji, Y., Bakiri, L., Qiu, Z., Cen, J., Chen, X., Chen, L., Scheuch, H., Zheng, H., Qin, L., et al. (2012). Liver cancer initiation is controlled by AP-1 through SIRT6-dependent inhibition of survivin. Nat Cell Biol 14, 1203-1211.

Mostoslavsky, R., Chua, K.F., Lombard, D.B., Pang, W.W., Fischer, M.R., Gellon, L., Liu, P., Mostoslavsky, G., Franco, S., Murphy, M.M., et al. (2006). Genomic instability and aging-like phenotype in the absence of mammalian SIRT6. Cell 124, 315-329.

Sebastian, C., Zwaans, B.M., Silberman, D.M., Gymrek, M., Goren, A., Zhong, L., Ram, O., Truelove, J., Guimaraes, A.R., Toiber, D., et al. (2012). The histone deacetylase SIRT6 is a tumor suppressor that controls cancer metabolism. Cell 151, 1185-1199.

Shah, R.B., Mehra, R., Chinnaiyan, A.M., Shen, R., Ghosh, D., Zhou, M., Macvicar, G.R., Varambally, S., Harwood, J., Bismar, T.A., et al. (2004). Androgen-independent prostate cancer is a heterogeneous 
group of diseases: lessons from a rapid autopsy program. Cancer Res 64, 9209-9216.

Song, N.Y., and Surh, Y.J. (2012). Janus-faced role of SIRT1 in tumorigenesis. Ann N Y Acad Sci 1271, 10-19.

Sundaresan, N.R., Vasudevan, P., Zhong, L., Kim, G., Samant, S., Parekh, V., Pillai, V.B., Ravindra, P.V., Gupta, M., Jeevanandam, V., et al. (2012). The sirtuin SIRT6 blocks IGF-Akt signaling and development of cardiac hypertrophy by targeting c-Jun. Nat Med 18, 1643-1650.

Taylor, B.S., Schultz, N., Hieronymus, H., Gopalan, A., Xiao, Y., Carver, B.S., Arora, V.K., Kaushik, P., Cerami, E., Reva, B., et al. (2010). Integrative genomic profiling of human prostate cancer. Cancer Cell 18, 11-22.

Van Meter, M., Mao, Z., Gorbunova, V., and Seluanov, A. (2011). SIRT6 overexpression induces massive apoptosis in cancer cells but not in normal cells. Cell Cycle 10, 3153-3158.
Xiao, C., Wang, R.H., Lahusen, T.J., Park, O., Bertola, A., Maruyama, T., Reynolds, D., Chen, Q., Xu, X., Young, H.A., et al. (2012). Progression of chronic liver inflammation and fibrosis driven by activation of cJUN signaling in Sirt6 mutant mice. J Biol Chem 287, 41903-41913.

Xie, Q.R., Liu, Y., Shao, J., Yang, J., Liu, T., Zhang, T., Wang, B., Mruk, D.D., Silvestrini, B., Cheng, C.Y., et al. (2013). Male contraceptive Adjudin is a potential anti-cancer drug. Biochem Pharmacol 85, 345-355.

Xie, X., Zhang, H., Gao, P., Wang, L., Zhang, A., Xie, S., and Li, J. (2012). Overexpression of SIRT6 in porcine fetal fibroblasts attenuates cytotoxicity and premature senescence caused by D-galactose and tert-butylhydroperoxide. DNA Cell Biol 31, 745-752.

Zhong, L., D'Urso, A., Toiber, D., Sebastian, C., Henry, R.E., Vadysirisack, D.D., Guimaraes, A., Marinelli, B., Wikstrom, J.D., Nir, T., et al. (2010). The histone deacetylase Sirt6 regulates glucose homeostasis via Hif1alpha. Cell 140, 280-293. 